\title{
Gedanke eines Ausbaues des Systems der Elemente auf breiterer Grundlage.
}

\author{
Von D. Bauarew.
}

Die Periodizität der Elemente tritt in der Veränderung ihrer chemischen Eigenschaften zutage. Diese Veränderung ist aber stark von dem Wechsel der Wertigkeit abhängig; es existieren Fälle, in welchen ein Element auf Grund der Eigenschaften der verschiedenen Reihen von Verbindungen, die es eingeht, verschiedene Plätze in dem periodischen System erhalten müBte. Typische Beispiele für ein solches Verhalten liefern $\mathrm{Cr}, \mathrm{Mn}, \mathrm{Cu}, \mathrm{Hg}$, Tl u. a. Das periodische System gibt solchen Elementen aber nur eine Stelle. Auf Grund des Obengesagten ist das ein wesentlicher Nachteil, der am einfachsten dadurch beseitigt wird, daB wir das periodische System, das die Elemente in einer Ebene darstellt, die mit der Druck- und Schreibfläche zusammenfällt, zu einem räumlichen System ausbauen, das in verschiedenen Richtungen betrachtet werden kann. Der Ausbau dieses räumlichen Systems würde sich am einfachsten in folgender Weise vollziehen.

Die räumliche Anordnung der Elemente lieB sich am besten durch Zuhilfenahme von Glasplatten verwirklichen, von denen wir 9 Stück benötigten. Auf jede dieser Glasplatten trugen wir an den Plätzen, die durch das ebene System vorgeschrieben werden, nur Elemente einer Wertigkeit ein, also auf die erste nur die Elemente der Nullgruppe, auf die zweite nur einwertige Elemente usw., dabei bleiben dann immer die Stellen der anderswertigen Elemente unbesetzt. Diese Glasplatten ordneten wir dann in gleichen Abständen parallel hintereinander an, und damit war das einfachste räumliche System gewonnen.

Worin bestehen nun die Vorzüge des neuen jetzt räumlichen periodischen Systems? 
Die Elemente sind darin nicht nur nach der Ordnungszahl ihrer Atomgewichte eingeordnet, sondern auch noch nach ihrer Wertigkeit. Jedes Element, von dem man weiB, daB es verschiedenwertig auftritt, ist jetzt dementsprechend auf verschiedenen Platten eingetragen. Dabei sind jeder Eintragung verschiedene Eigenschaften des Elementes zugeordnet, die von der betreffenden Wertigkeit abhängen. Solche Eigenschaften des betreffenden Elementes ändern sich sprunghaft wie die Wertigkeit. Die so erreichte Anordnung nach der Wertigkeit ist ein Vorzug des räumlichen Systems vor dem ebenen und entspricht den heutigen Anschauungen rom Wesen der Wertigkeit.

Trotzdem dieses System räumlich ist, hat es die Anordnung nach den Ordnungszahlen der Atomgewichte beibehalten, die Ansicht senkrecht auf die Platten liefert uns das ebene periodische System, alle Eintragungen fallen wieder in eine Ebene, die Bildebene, mehrfache Eintragungen eines Elementes kommen zur Deckung. Damit vereint das neue System alle Vorzüge der MENDELEJEwschen Anordnung in sich. Diese Anordnung erlaubt uns auch diejenigen Eigenschaften der Elemente miteinander zu vergleichen, die nach unsrer gegenwärtigen Kenntnis rom Aggregatzustand und nicht von der Wertigkeit der Atome abhängen. Solche Eigenschaften sind z. B. Dichte, Atomvolumen, Schmelzpunkte, Siedepunkte usw., überhaupt die physikalischen Eigenschaften der festen und flüssigen Elemente.

Der wichtigste Vorzug des so geschaffenen räumlichen Systems bleibt aber der, daB in ihm die meisten der Elemente auf verschiedenen Platten einzutragen sind, entsprechend ihrer verschiedenen Wertigkeit. Es ist bekannt, daB Elemente, die im periodischen System einander benachbart sind, in den Verbindungen, wo sie gleiche Wertigkeit zeigen, sich ähnlich verhalten. Es gilt dies z. B. für $\mathrm{Cu}^{\mathrm{I}}, \mathrm{Ag}^{\mathrm{I}}$ und $\mathrm{Au}^{\mathrm{I}}$; $\mathrm{Cu}^{\mathrm{II}}, \mathrm{Zn}^{\mathrm{II}}, \mathrm{Cd}^{\mathrm{II}}$ und $\mathrm{Hg}^{\mathrm{II}}$; $\mathrm{Cr}^{\cdots \cdot}, \mathrm{Fe}^{\cdots \cdot}$ und $\mathrm{Al}^{\cdots *}$; $\mathrm{S}^{\mathrm{vI}}$ und $\mathrm{Cr}^{\mathrm{VI}}, \mathrm{Mn}^{\mathrm{VII}}$ und $\mathrm{Cl}^{\mathrm{vII}}$ usw. Dieser Ähnlichkeit ist in dem neuen periodischen System nun glänzend Rechnung getragen worden. Damit ist eine Darstellung des Verhaltens der Elemente in verschiedenen Teilreihen erreicht, die ihre anschauliche Wiedergabe auf verschiedenen Platten findet. Dabei bemerkt man, dab die auf einer Platte vorhandenen RegelmäBigkeiten, sich von Platte zu Platte ändern, einander aber immer ähnlich bleiben.

Im räumlichen System ändern sich die Eigenschaften der Elemente sprungweise sowohl auf derselben Platte als auch beim Ühergang von einer Platte zur andern und nun finden wir nicht nur 
24 D. Balarew. Aufbau des Systems der Elemente auf breiterer Grundlage.

RegelmäBigkeiten in den Spalten und Reihen wie im ebenen System, sondern auch noch in den hintereinanderliegenden Kolonnen.

Besonders klar treten in diesem räumlichen System RETGEns Resultate bezüglich des Isomorphismus der entsprechenden Verbindungen der verschiedenen Elemente zutage.

Das räumliche System läBt auch klarer den Wernerschen Gedanken begreifen, daß die meisten der Elemente mit ihrer Hauptralenz auch in fast alle Verbindungen mit niedriger Valenz eingehen. Das hier in groBen Zügen entwickelte neue System wejst also nicht nur für noch nicht entdeckte chemische Elemente, sondern auch für noch nicht entdeckte Wertigkeiten der meisten Elemente Lüicken auf.

Sofla, Universität.

Bei der Redaktion eingegangen am 14. November 1921. 